\title{
Imaging Characteristics of Pediatric Diffuse Midline Gliomas with Histone H3 K27M Mutation
}

\author{
(D) M.S. Aboian, DD.A. Solomon, (DE. Felton, (DM.C. Mabray, DJ.E. Villanueva-Meyer, (D) S. Mueller, and (DS. Cha
}

\begin{abstract}
BACKGROUND AND PURPOSE: The 2016 World Health Organization Classification of Tumors of the Central Nervous System includes "diffuse midline glioma with histone H3 K27M mutation" as a new diagnostic entity. We describe the MR imaging characteristics of this new tumor entity in pediatric patients.
\end{abstract}

MATERIALS AND METHODS: We retrospectively reviewed imaging features of pediatric patients with midline gliomas with or without the histone H3 K27 mutation. We evaluated the imaging features of these tumors on the basis of location, enhancement pattern, and necrosis.

RESULTS: Among 33 patients with diffuse midline gliomas, histone H3 K27M mutation was present in 24 patients (72.7\%) and absent in 9 (27.3\%). Of the tumors, $27.3 \%(n=9)$ were located in the thalamus; $42.4 \%(n=14)$, in the pons; $15 \%(n=5)$, within the vermis/fourth ventricle; and $6 \%(n=2)$, in the spinal cord. The radiographic features of diffuse midline gliomas with histone H3 K27M mutation were highly variable, ranging from expansile masses without enhancement or necrosis with large areas of surrounding infiltrative growth to peripherally enhancing masses with central necrosis with significant mass effect but little surrounding T2/FLAIR hyperintensity. When we compared diffuse midline gliomas on the basis of the presence or absence of histone H3 K27M mutation, there was no significant correlation between enhancement or border characteristics, infiltrative appearance, or presence of edema.

CONCLUSIONS: We describe, for the first time, the MR imaging features of pediatric diffuse midline gliomas with histone H3 K27M mutation. Similar to the heterogeneous histologic features among these tumors, they also have a diverse imaging appearance without distinguishing features from histone $\mathrm{H} 3$ wildtype diffuse gliomas.

T 2016 World Health Organization Classification of Tumors of the Central Nervous System reorganizes diffuse astrocytic tumors on the basis of their genetic alterations in addition to histopathologic features, with inclusion of a new entity, the "dif-

\footnotetext{
Received September 8, 2016; accepted after revision November 6.

From the Department of Radiology (M.S.A., E.F., M.C.M., J.E.V.-M., S.C.); Division of Neuropathology (D.A.S.), Department of Pathology; Division of Pediatric Hematology/Oncology (S.M.), Department of Pediatrics; Department of Neurological Surgery (S.M.); and Division of Child Neurology (S.M.), Department of Neurology, University of California, San Francisco, San Francisco, California.

D.A.S. is supported by the National Institutes of Health Director's Early Independence Award (DP5 OD021403) and Career Development Award from the University of California, San Francisco, Brain Tumor Specialized Programs of Research Excellence (P50 CA097257). S.M. is supported by the National Center for Advancing Translational Sciences, National Institutes of Health, through University of California, San Francisco-Clinical and Translational Science Institute grant No. KL2TR000143. M.S.A. and J.E.V.-M. are supported by the National Institutes of Health Biomedical Imaging and Bioengineering T32 grant (No. 5T32EB001631-12). Please address correspondence to Soonmee Cha, MD, Department of Radiology, University of California, San Francisco, 505 Parnassus Ave, San Francisco, CA 94143; e-mail: soonmee.cha@ucsf.edu

- Indicates open access to non-subscribers at www.ajnr.org

http://dx.doi.org/10.3174/ajnr.A5076
}

fuse midline glioma, histone H3 K27M mutant." ${ }^{11}$ The histone H3 $\mathrm{K} 27 \mathrm{M}$ missense mutation is present in most adult and pediatric diffuse gliomas arising in midline CNS structures, including the thalamus, hypothalamus, third ventricle, pineal region, cerebellum, brain stem, and spinal cord. ${ }^{2-6}$ The K27M mutation occurs in either of 2 genes, H3F3A or HIST1H3B, which encode the histone $\mathrm{H} 3$ variants, $\mathrm{H} 3.3$ and $\mathrm{H} 3.1$, respectively. ${ }^{7,8}$ While the K27M mutation appears quite specific for diffuse gliomas arising in midline structures, a separate missense variant (G34R or G34V) in $H 3 F 3 A$ can sometimes be found within peripheral cerebral hemispheric gliomas predominantly in teenage and young adult patients. ${ }^{2,3,8}$ These mutations result in decreased methylation of the histone tails, resulting in altered gene expression patterns thought to block glial differentiation and promote gliomagenesis. ${ }^{2,3}$

Here, we characterize the imaging features of this new tumor entity, diffuse midline gliomas with histone H3 K27M mutation. We also compare the imaging characteristics of histone H3 K27M mutant and wildtype diffuse midline gliomas to determine whether 
any specific imaging features correlate with histone H3 K27M mutational status.

\section{MATERIALS AND METHODS}

We identified 33 pediatric patients with diffuse midline gliomas that were tested for the presence of the histone H3 K27M mutation and underwent primary work-up or tumor board review at our institution. This retrospective study was performed in compliance with Health Insurance Portability and Accountability Act regulations and was approved by our institutional review board.

Formalin-fixed, paraffin-embedded tumor tissue was analyzed by a University of California, San Francisco neuropathologist (D.A.S.). Immunohistochemistry for histone H3 K27M mutant protein was performed by using a rabbit polyclonal antibody (ABE419; EMD Milipore, Billerica, Massachusetts), which detects histone H3.3 and H3.1 tails, as previously described. ${ }^{6,9}$ Immunohistochemical staining was performed in a BenchMark XT Auto Stainer (Ventana Medical Systems, Tucson, Arizona) by using the Cell Conditioning 1 (Ventana Medical Systems) antigen retrieval buffer for 30 minutes at $95^{\circ} \mathrm{C}$, incubation with a primary antibody at 1:500 dilution for 32 minutes, and the ultraView Universal DAB Detection Kit (Ventana Medical Systems).

All patients underwent MR imaging of the brain on either 1.5T or 3T clinical scanners by using the following protocol: 3-plane localizer, axial T2-weighted (TR/TE, 2500/80 ms), 3D fluid-attenuated inversion recovery (TR/TE/TI, 5500/136/2200 ms), T1weighted (TR/TE, 10/4 ms) without and with and intravenous gadolinium, and axial diffusion-weighted imaging (TR/TE, 7000/60 ms) sequences.

Pretreatment MR imaging of each patient was reviewed by a neuroradiology attending physician (S.C.) and a neuroradiology fellow (M.S.A.) for the following tumor characteristics: location, size, multifocality, involvement of the subventricular zone, cortical invasion, pattern of contrast enhancement, solid or cystic components, necrosis, edema, infiltrative pattern, mass effect, and border characteristics. Type of tumor spread (local or distant) was also characterized on follow-up MRIs if available. Comparative analysis of imaging-pattern difference between histone $\mathrm{H} 3$ K27M wildtype and mutant diffuse gliomas with the Pearson correlation coefficient was performed. Statistical analysis was performed on STATA (StataCorp, College Station, Texas).

\section{RESULTS}

Patient age, tumor location, and histone H3 K27M mutant protein status for the 33 patients with diffuse midline gliomas included in this study are shown in Table 1 . The histone H3 $\mathrm{K} 27 \mathrm{M}$ mutant protein was found in 24 of the 33 (72.7\%) studied tumors.

Long-term follow-up was available in 21 patients, with time of resection/biopsy to date of death available for 10 patients, including 2 patients with K27M mutant cervical spine tumors (10.4 and 17.4 months), 4 patients with K27M mutant pontine tumors (5.116.5 months), 1 of 3 deceased patients with K27M mutant vermian/fourth ventricle tumors (7.5 months), 2 patients with K27M mutant thalamic tumors (5.6-14.5 months), and 1 patient with histone H3 wildtype pontine tumor (9.6 months). Both of the
Table 1: Patient demographics ${ }^{a}$

\begin{tabular}{lccc}
\hline & $\begin{array}{c}\text { All } \\
\text { Patients } \\
(\boldsymbol{n}=\mathbf{3 3})\end{array}$ & $\begin{array}{c}\text { Histone H3 } \\
\text { K27M Mutant } \\
(\boldsymbol{n}=\mathbf{2 4})\end{array}$ & $\begin{array}{c}\text { Histone H3 } \\
\text { Wildtype } \\
(\boldsymbol{n}=\mathbf{9})\end{array}$ \\
\hline Age & & & \\
$\quad$ Mean & $115.8 \mathrm{mo}$ & $108.5 \mathrm{mo}$ & $135.2 \mathrm{mo}$ \\
Range & $8.2-232 \mathrm{mo}$ & $32-232 \mathrm{mo}$ & $8.2-211 \mathrm{mo}$ \\
Sex & & & \\
Male & 25 & 17 & 8 \\
Female & 8 & 7 & 1 \\
Anatomic location & & & 0 \\
Subcallosal & 1 & 1 & 3 \\
Thalamus & 9 & 6 & 2 \\
Midbrain tectum & 2 & 0 & 3 \\
Pons & 14 & 11 & 1 \\
Vermis/fourth ventricle & 5 & 4 & 0 \\
Cervical spine & 2 & 2 & \\
\hline
\end{tabular}

a Data are number and age.

patients with tectal tumors are presently alive at 23.3 and 78 months' follow-up after diagnosis. There were 11 total living patients on follow-up. Short-term imaging follow-up was available in 7 patients, but due to lack of clinical follow-up, these were not included in the analysis.

\section{Diffuse Midline Gliomas Centered within the Posterior Fossa}

Nineteen patients had infratentorial tumors, with 14 centered within the pons and 5 located within the vermis/fourth ventricle (Fig 1). Of these patients, 11/14 (78.6\%) pontine tumors were positive for histone H3 K27M mutant protein and 4/5 (80\%) of the vermis/fourth ventricle tumors were positive for histone $\mathrm{H} 3$ K27M mutant (Table 1).

Histone H3 K27M mutant tumors were centered within either the pons or cerebellar peduncle, and most demonstrated contrast enhancement (67\%) (Fig 1 and Table 2). Five tumors were centered within the vermis/fourth ventricle and extended into the posterior pons, and they demonstrated uniform contrast enhancement. Ten tumors were centered within the pons and had heterogeneous enhancement characteristics (Fig 1 and Table 2). Two patients with histone H3 K27M mutant posterior fossa tumors had a multifocal appearance at presentation.

Posterior fossa histone $\mathrm{H} 3$ wildtype diffuse midline gliomas $(n=4)$ were heterogeneous in their imaging characteristics (Table 2). One of these tumors was centered within the subependymal surface of the cerebellar hemisphere and was extending into the fourth ventricle, resulting in hydrocephalus. Another tumor was centered predominantly within the left cerebellar hemisphere with minimal extension into the fourth ventricle and no associated hydrocephalus. The third patient's tumor was centered within the pons with extension into the cerebral peduncles and into the right posterior limb of the internal capsule. This tumor demonstrated mild central enhancement and greatly resembled the histone $\mathrm{H} 3 \mathrm{~K} 27 \mathrm{M}$ mutant tumors.

Of 15 posterior fossa gliomas with histone H3 K27M mutation, 14 patients had follow-up that ranged from 3 to 32 months. Ten patients developed tumor recurrence within the treatment bed at 3.8-18.4 months of follow-up. Two of these patients demonstrated CSF-based spread within 10.7 and 4.9 months of follow-up. Only 4 of these patients had no tumor progression on 


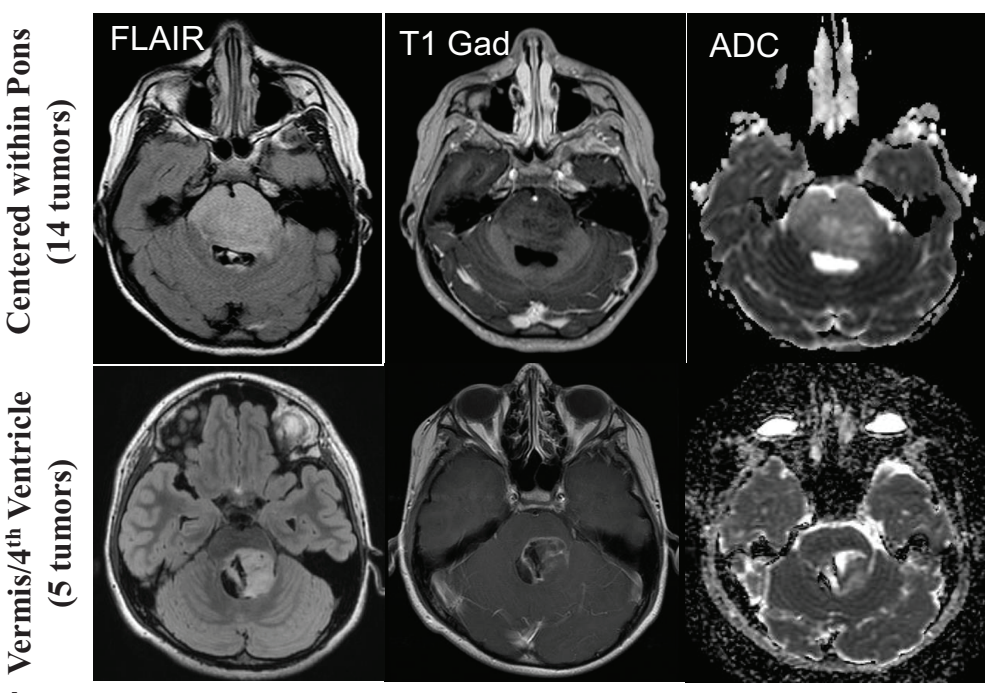

A

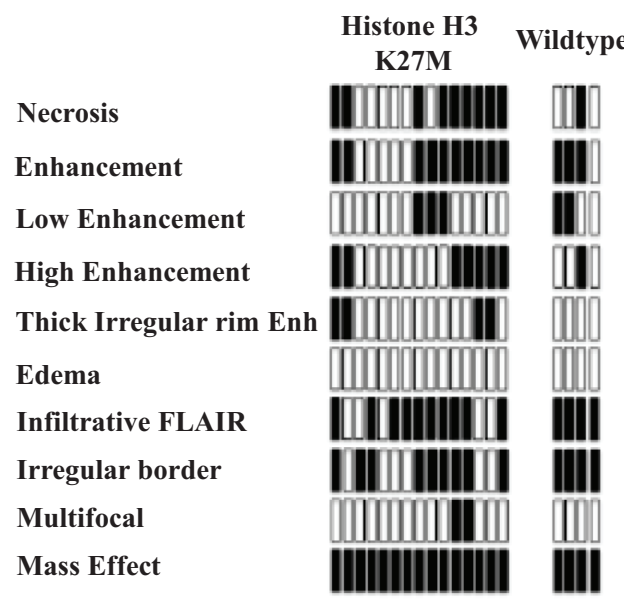

B

FIG 1. Diffuse midline gliomas within the posterior fossa. A, FLAIR, T1 postgadolinum, and ADC maps of infratentorial diffuse gliomas demonstrate 2 distinct appearances, with the first type being centered within the pons with expansion of the pons and engulfment of the basilar artery (14 patients). The second type was centered within the vermis and cerebellar peduncle with extension into the fourth ventricle and involvement of the posterior pons (5 patients). $B$, Imaging features of both the histone H3 K27M and wildtype tumors were heterogeneous with respect to necrosis, patterns of enhancement, edema, and infiltrative features. Black bars represent the presence of the feature; white bars represent lack of the feature.

Table 2: MRI characteristics ${ }^{\mathrm{a}}$

\begin{tabular}{lccc}
\hline & $\begin{array}{c}\text { All } \\
\text { Patients } \\
(\boldsymbol{n}=\mathbf{3 3})\end{array}$ & $\begin{array}{c}\text { Histone H3 } \\
\text { K27 Mutant } \\
(\boldsymbol{n}=\mathbf{2 4})\end{array}$ & $\begin{array}{c}\text { Histone H3 } \\
\text { Wildtype } \\
(\boldsymbol{n}=\mathbf{9})\end{array}$ \\
\hline Multifocality & 5 & 5 & 0 \\
Contrast enhancement & 22 & 16 & 6 \\
$\begin{array}{l}\text { Cystic component or } \\
\quad \text { necrosis }\end{array}$ & 18 & 15 & 3 \\
Edema & 4 & 4 & 0 \\
Infiltrative pattern & 27 & 18 & 9 \\
Mass effect & 32 & 24 & 8 \\
Irregular border & 27 & 18 & 9 \\
CSF-based metastases & 7 & 6 & 1 \\
Direct cortical invasion & 12 & 9 & 3 \\
\hline
\end{tabular}

a Data are number.

follow-up, ranging from 3 to 8.5 months. In histone $\mathrm{H} 3$ wildtype pontine gliomas $(n=4), 2$ patients demonstrated local progression at 4.9 and 9.5 months, while 1 patient developed distal metastatic disease at 3.3 months of follow-up. One of the patients with a histone $\mathrm{H} 3$ wildtype glioma did not have available follow-up. Aggressive patterns of tumor progression, including local infiltrative tumor growth and distal CSF-based tumor spread, were seen in both histone H3 K27M mutant and wildtype tumors (Figs 2 and 3).

\section{Comparison of Histone H3 K27M Mutant and Wildtype Posterior Fossa Diffuse Midline Gliomas}

No significant difference on imaging at presentation was noted between the histone $\mathrm{H} 3 \mathrm{~K} 27 \mathrm{M}$ mutant and wildtype posterior fossa tumors. There were only 4 histone $\mathrm{H} 3$ wildtype posterior fossa tumors; therefore, comparison with the K27M mutant tumors $(n=15)$ is limited. Overall, the K27M mutant tumors were centered in either the pons or vermis/fourth ventricle, with extension into the pons. Fourth ventricle/vermian K27M mutant tumors were mostly enhancing (4/5), while tumors centered within the pons were heterogeneous in appearance in regard to their enhancement characteristics. Two of the K27M mutant tumors centered within the pons were multifocal, with an additional focus of an infiltrative masslike FLAIR hyperintense lesion within the cerebellar hemisphere. The histone $\mathrm{H} 3$ wildtype tumors in the posterior fossa were also located within the pons, the vermis with extension into the fourth ventricle, and the cerebellar hemisphere. Three of the 4 wildtype tumors demonstrated enhancement at diagnosis. Imaging comparison of the histone $\mathrm{H} 3$ wildtype with K27M mutant tumors is shown in Fig 1, demonstrating no significant differences between the 2 groups.

\section{Diffuse Midline Gliomas Centered within the Thalamus}

In 9 patients, tumors were centered within the thalamus, with 6 of these patients having histone $\mathrm{H} 3 \mathrm{~K} 27 \mathrm{M}$ mutations. Of the patients having histone $\mathrm{H} 3 \mathrm{~K} 27 \mathrm{M}$ mutations, 3 had contrast enhancement of the tumor and 3 did not (Fig 4). One of the patients had tumor involving the bilateral thalami, and imaging did not demonstrate contrast enhancement. Of the patients with wildtype tumors, 1 had prominent enhancement, 1 had punctate enhancement, and 1 did not have preoperative imaging (data not included).

Four of the patients with K27M mutant thalamic gliomas had follow-up, with 2 patients demonstrating distant CSF-based metastatic disease and 1 having local recurrence (images not shown). One of the patients did not have any progression at 5.8 months' follow-up. None of the patients with wildtype histone H3 had clinical follow-up to ascertain recurrent disease.

Comparison of histone H3 K27M mutant and wildtype tumors centered within the thalamus is limited due to low numbers, with 6 patients having $\mathrm{K} 27 \mathrm{M}$ mutation and 3 with wildtype histone $\mathrm{H} 3$. This limited comparison did not identify any specific features that differentiated the K27M mutant from wildtype tumors. 


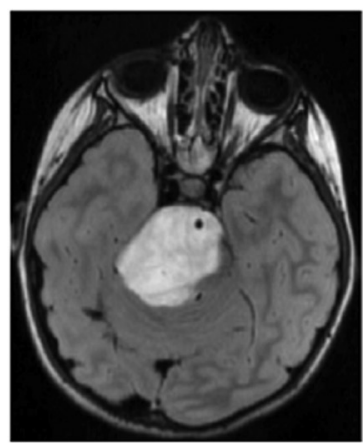

A
Presentation scan

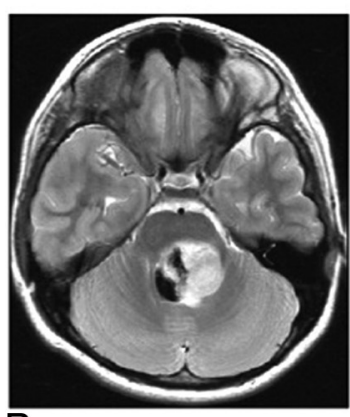

B

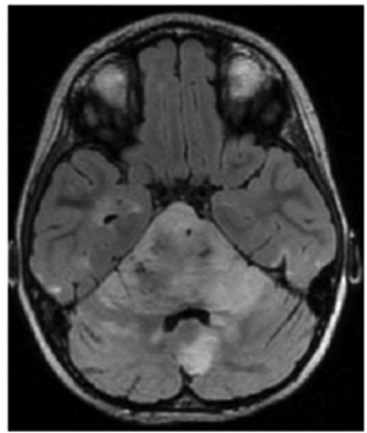

Local infiltrative and distal CSF based spread (10.7 months)
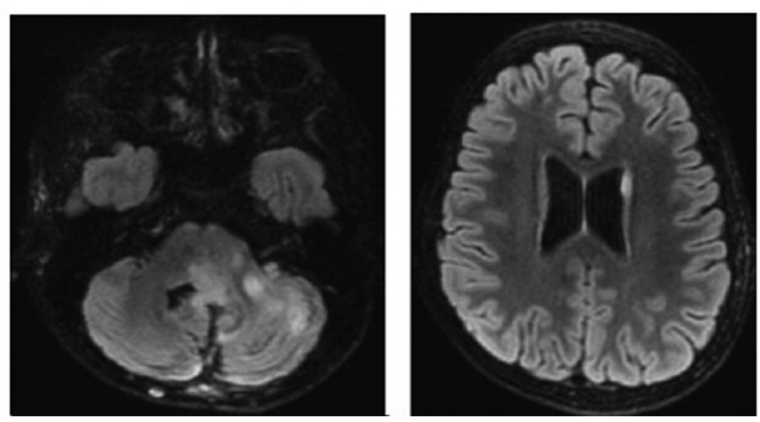

Local infiltrative spread (25.7 months)

FIG 2. Imaging of tumor progression in histone $\mathrm{H} 3 \mathrm{~K} 27 \mathrm{M}$ mutant tumors. FLAIR and T1-weighted contrast-enhancing images demonstrate local infiltrative $(A)$ and CSF-based progression $(B)$ in histone $\mathrm{H} 3 \mathrm{~K} 27 \mathrm{M}$ mutants.
Presentation scan

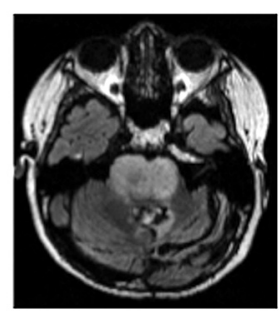

Presentation scan

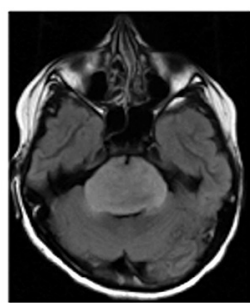

Mostly CSF based metastatic disease with evidence of local progression within pons

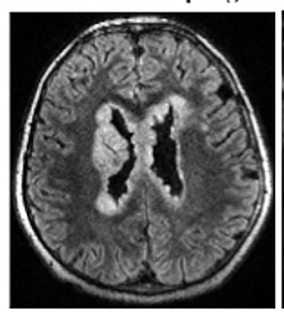

Local progression

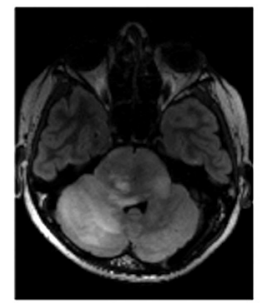

FIG 3. Imaging of tumor progression in histone $\mathrm{H} 3$ wildtype tumors. FLAIR and T1-weighted contrast-enhancing images demonstrate mixed local infiltrative progression with CSF-based metastatic disease $(A)$ and local infiltrative progression $(B)$.

\section{Other Midline Locations for Diffuse Midline Gliomas: Subcallosal Area and Tectum}

One patient with subcallosal diffuse glioma was identified. This tumor was strongly enhancing and had regions of reduced diffusion. The patient underwent gross total resection and did not have evidence of tumor progression after 3.8 months of follow-up.
In addition, 2 patients had diffuse gliomas centered within the tectum. Both of these patients were alive at 23.3 and 78 months' follow-up after biopsy. On contrast administration, these tumors were heterogeneous, with 1 tumor demonstrating contrast enhancement and a second tumor being nonenhancing. Both patients presented with symptoms due to hydrocephalus.

\section{Diffuse Midline Gliomas of the Cervical Spinal Cord}

Cervical spine tumors had expansile FLAIR hyperintense signal within the spinal cord with evidence of internal enhancement and reduced diffusion on initial imaging (Fig 5). One of the patients had FLAIR hyperintense expansile regions within the folia of the cerebellar hemispheres at the time of initial imaging, which was suspicious for metastatic spread via the subependymal route (Fig 5). The second patient presented with tumor localized to the cervical cord but developed subependymal metastatic disease to the posterior fossa subependymal region of the fourth ventricle approximately 5 months after the original scan (data not shown).

\section{DISCUSSION}

The 2016 CNS World Health Organization classification introduced a new entity of "diffuse glioma with histone H3 K27M mutation." In this study, we describe the MR imaging features of diffuse midline gliomas with respect to their appearance within the thalamus, pons, vermis/cerebellum, cervical spine, and other sites based on histone H3 K27M mutation status.

The presence of the histone H3 K27M mutation results in decreased methylation of histone tails of the histone $\mathrm{H} 3$ family proteins, mainly H3.1 and H3.3. Previous studies demonstrated that in patients with diffuse intrinsic pontine gliomas with lysine 27 mutation in the $\mathrm{H} 3.3$ protein tail, overall survival was worse compared with H3.1-mutated subgroup. ${ }^{8}$ Statistical modeling demonstrated that the type of histone carrying the $\mathrm{K} 27 \mathrm{M}$ mutation was the most important predictive factor of overall survival in this group of patients, while MR imaging criteria of contrast enhancement alone did not play a significant role in predicting overall survival. ${ }^{8}$ In addition, Jansen et $\mathrm{al}^{10}$ reported the overall risk score of new diffuse intrinsic pontine gliomas, which depends on patient age, symptom duration, treatment type, and radiologic presence of contrast ring enhancement within diffuse intrinsic pontine gliomas. Our analysis is based on clinical assessment of the presence of histone $\mathrm{H} 3$ mutation status with immunohistochemistry as described by the new World Health Organization criteria. ${ }^{1}$ This assessment does not distinguish H3.1 from H3.3 mutation but 


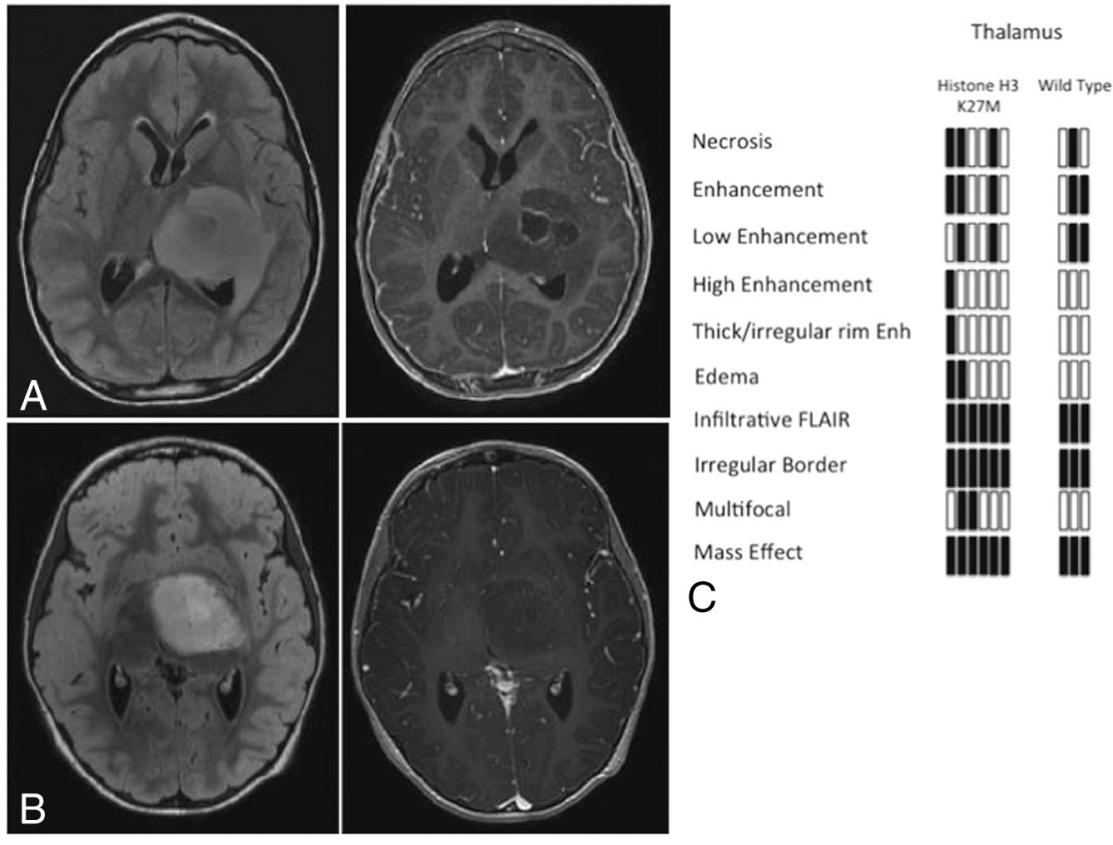

FIG 4. Imaging of midline gliomas centered within the thalamus. Midline gliomas with histone $\mathrm{H} 3$ K27M mutation centered within the thalamus had 2 specific imaging presentations, with $(A)$ and without $(B)$ contrast enhancement. Three patients had contrast enhancement, and 3 patients did not. $C$, Imaging features within the thalamic gliomas based on the presence of the histone $\mathrm{H} 3$ K27M mutation. Black bars represent the presence of the feature; white bars represent lack of the feature.
$67 \%$ of patients, and cervical spine gliomas being uniformly enhancing. Cervical spine gliomas with histone $\mathrm{H} 3$ K27M mutation demonstrated prominent CSF-based metastatic spread, while thalamic and pontine gliomas demonstrated a variety of progressive patterns, most commonly local recurrence.

Comparison of the histone H3 K27M mutant glioma with the wildtype is limited due to the infrequent occurrence of histone $\mathrm{H} 3$ wildtype diffuse gliomas within the midline in pediatric patients. In our series, we had only 3 thalamic and 4 pontine histone $\mathrm{H} 3$ wildtype diffuse gliomas. In addition, evaluation of diffuse gliomas based on tumor location is limited due to the low number of representative tumors in the thalamus, ${ }^{9}$ tectum, ${ }^{2}$ subcallosal region, ${ }^{1}$ and cervical spine. ${ }^{2}$ This limited analysis did not identify differentiating features based on imaging characteristics that would help distinguish histone H3 K27M mutant from wildtype tumors.

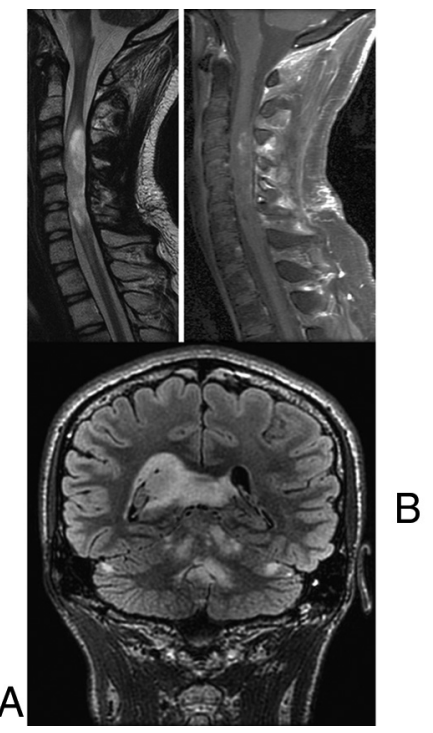

FIG 5. Imaging of cervical spine histone H3 K27M mutant gliomas. T2-weighted and postcontrast T1-weighted imaging of cervical spine glioblastomas that presented with subependymal metastatic disease and evidence of significant progression involving the subependymal surfaces of the lateral ventricles and the folia of the cerebellum at 5 months after initial diagnosis.

defines a subgroup of midline gliomas with poor clinical prognosis. This provides an important clinical context to interpretation of clinical pathology reports. ${ }^{11,12}$

Imaging features of histone H3 K27M mutant gliomas were heterogeneous, with thalamic gliomas demonstrating contrast enhancement and necrosis in 50\% of patients, pontine gliomas demonstrating contrast enhancement to a variable degree in

\section{CONCLUSIONS}

Our MR imaging characteristics of pediatric diffuse midline gliomas are based on histone H3 K27M mutational status. We found that midline gliomas with histone H3 K27M mutation centered within the thalamus and posterior fossa were more likely to be solid or infiltrative with infrequent necrosis. Tumor progression patterns between histone $\mathrm{H} 3 \mathrm{~K} 27 \mathrm{M}$ and wildtype tumors were similar, with local infiltrative tumor growth and distal CSF-based metastatic disease. Limited evaluation of 2 spinal cord diffuse gliomas with H3 K27M mutation showed distant and early metastasis to the brain.

\section{REFERENCES}

1. Louis DN, Perry A, Reifenberger G, et al. The 2016 World Health Organization Classification of Tumors of the Central Nervous System: a summary. Acta Neuropathol 2016;131:803-20 CrossRef Medline

2. Fontebasso AM, Liu XY, Sturm D, et al. Chromatin remodeling defects in pediatric and young adult glioblastoma: a tale of a variant histone 3 tail. Brain Pathol 2013;23;210-16 CrossRef Medline

3. Fontebasso AM, Schwartzentruber J, Khuong-Quang DA, et al. Mutations in SETD2 and genes affecting histone H3K36 methylation target hemispheric high-grade gliomas. Acta Neuropathol 2013;125: 659-69 CrossRef Medline

4. Hashizume R, Andor N, Ihara Y, et al. Pharmacologic inhibition of histone demethylation as a therapy for pediatric brainstem glioma. Nat Med 2014;20:1394-96 CrossRef Medline

5. Schwartzentruber J, Korshunov A, Liu XY, et al. Driver mutations in histone $\mathrm{H} 3.3$ and chromatin remodelling genes in paediatric glioblastoma. Nature 2012;482:226-31 CrossRef Medline

6. Solomon DA, Wood MD, Tihan T, et al. Diffuse midline gliomas with histone $\mathrm{H} 3-\mathrm{K} 27 \mathrm{M}$ mutation: a series of 47 cases assessing the

AJNR Am J Neuroradiol 38:795-800 Apr 2017 www.ajnr.org

799 
spectrum of morphologic variation and associated genetic alterations. Brain Pathol 2016;26:569-80 CrossRef Medline

7. Wu G1, Broniscer A, McEachron TA, et al; St. Jude Children's Research Hospital-Washington University Pediatric Cancer Genome Project. Somatic histone $\mathrm{H} 3$ alterations in pediatric diffuse intrinsic pontine gliomas and non-brainstem glioblastomas. Nat Genet 2012; 44:51-53 CrossRef Medline

8. Castel D, Philippe C, Calmon R, et al. Histone H3F3A and HIST1H3B K27M mutations define two subgroups of diffuse intrinsic pontine gliomas with different prognosis and phenotypes. Acta Neuropathol 2015;30:815-27 CrossRef Medline

9. Bechet D, Gielen GG, Korshunov A, et al. Specific detection of methionine 27 mutation in histone 3 variants (H3K27M) in fixed tis- sue from high-grade astrocytomas. Acta Neuropathol 2014;128: 733-41 CrossRef Medline

10. Jansen MH, Veldhuijzen van Zanten SE, Sanchez Aliaga E, et al. Survival prediction model of children with diffuse intrinsic pontine glioma based on clinical and radiological criteria. Neuro Oncol 2015; 17:160-66 CrossRef Medline

11. Jones C, Perryman L, Hargrave D. Paediatric and adult malignant glioma: close relatives or distant cousins? Nat Rev Clin Oncol 2012; 9:400-13 CrossRef Medline

12. Buczkowicz P, Bartels U, Bouffet E, et al. Histopathological spectrum of paediatric diffuse intrinsic pontine glioma: diagnostic and therapeutic implications. Acta Neuropathol 2014;128:573-81 CrossRef Medline 